Supporting Information for

\title{
Crystalline Semiconductor Boron Quantum Dots
}

Jinqian Hao, ${ }^{\dagger}$ Guoan Tai, ${ }^{* \dagger}$, Jianxin Zhou, ${ }^{\dagger}$ Rui Wang, ${ }^{\dagger,}+$ Chuang Hou, ${ }^{\dagger}$ and Wanlin Guo,$\dagger$

$\dagger$ The State Key Laboratory of Mechanics and Control of Mechanical Structures, Laboratory of Intelligent Nano Materials and Devices of Ministry of Education, College of Aerospace Engineering, Nanjing University of Aeronautics and Astronautics, Nanjing 210016, China.

* School of Material Science and Technology, Nanjing University of Aeronautics and Astronautics, Nanjing 210016, China.

\section{Corresponding Authors}

E-mail: taiguoan@nuaa.edu.cn

E-mail:wlguo@nuaa.edu.cn 

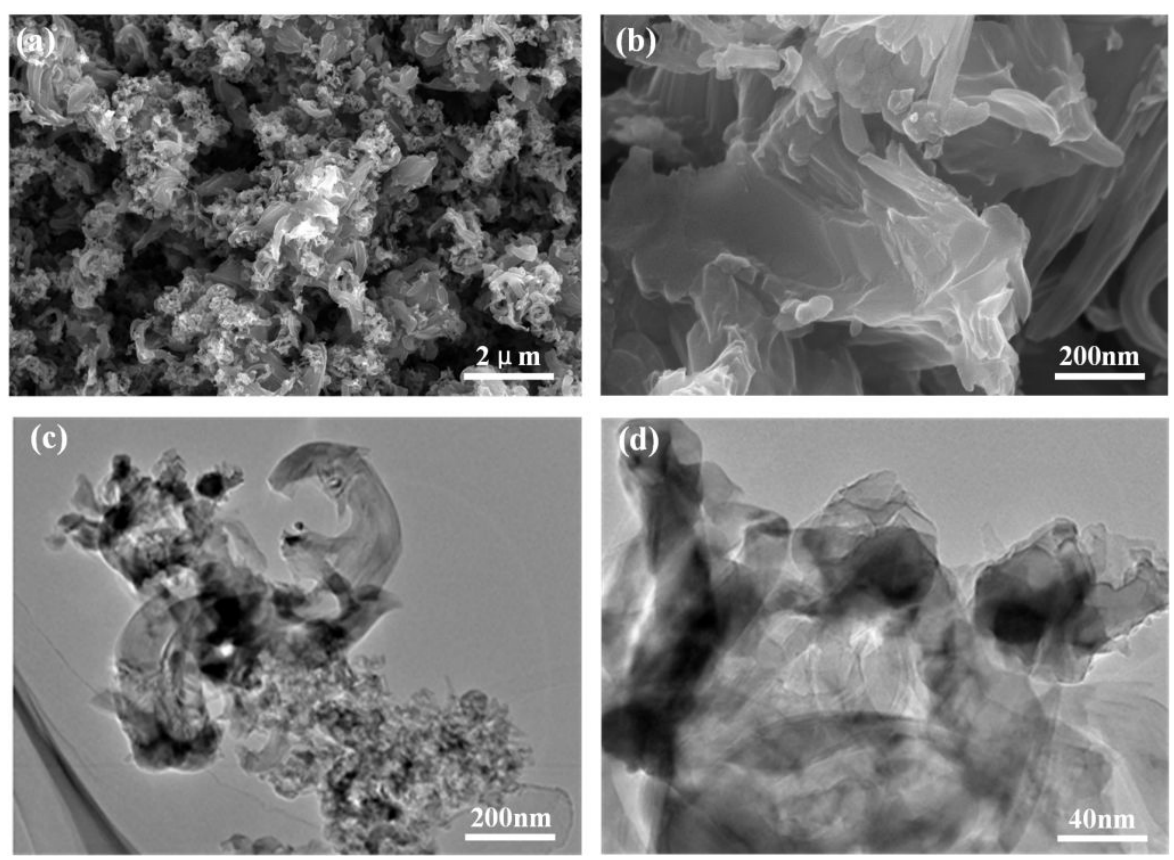

Figure S1. SEM and TEM images of the expanded boron powder. (a) Low-magnification SEM image of the powder. (b) High-magnification SEM image of the powder, indicating that the boron powder has a layered-curl structure. (c) Low-magnification TEM image of the powder. (d) Further magificated TEM image of the powder, suggesting that the powder has a layer feature. 

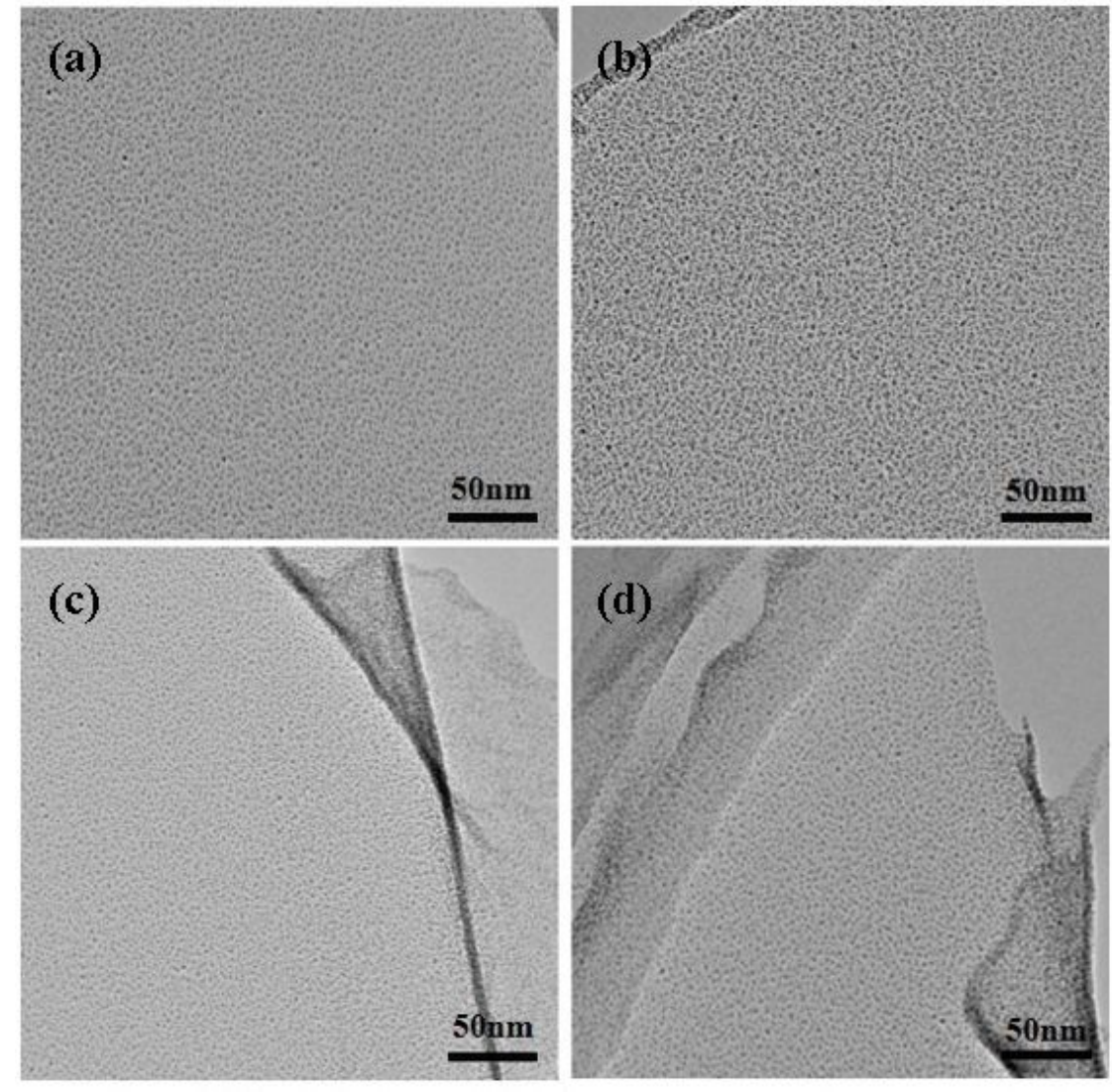

Figure S2. TEM images of the quantum dots in different batches.

To further address the reproducibility of material preparation, we also provide TEM images of the quantum dots (BQDs) successfully synthesized in four different batches, as shown in Figure S2. The BQDs were first observed under a high-resolution field emission transmission electron microscope at the end of 2018 (Figure S2a). During this process, it took us almost one year to determine the optimal preparation condition. Meanwhile, the experiment was successfully repeated many times under the optimal condition, as shown in Figure S2(b-d). The BQDs can be clearly observed at the edges of the cracked carbon films on the copper meshes. 


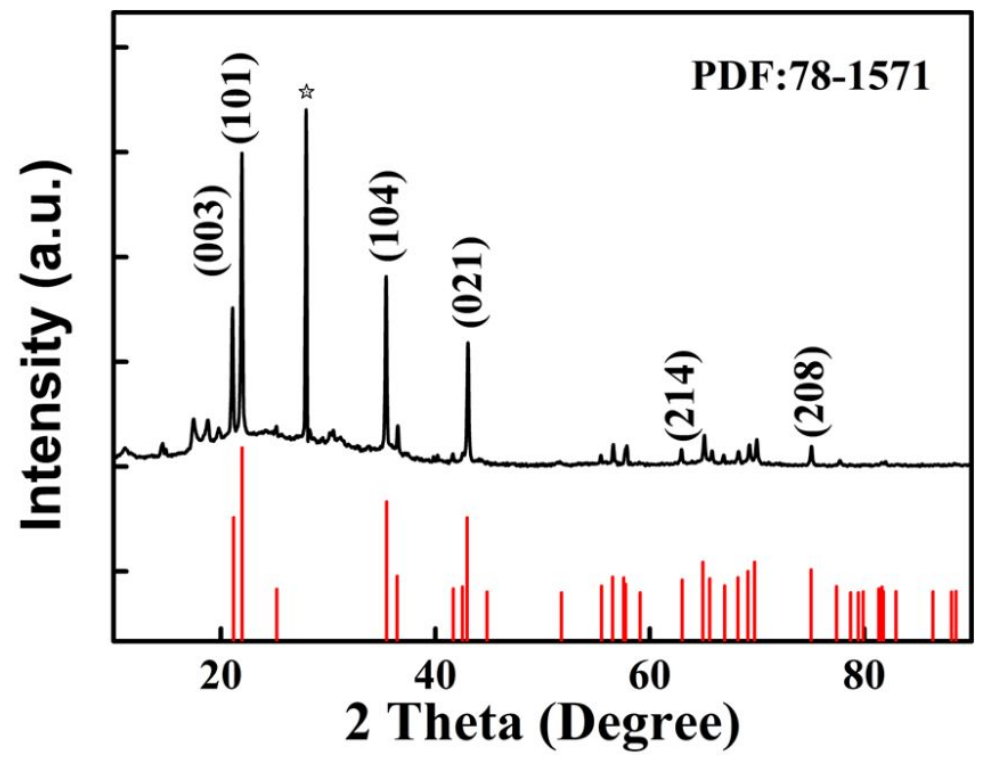

Figure S3. The XRD spectrum of the boron powder. The peak marked as "*” are from carbon (PDF: 22-1069). 


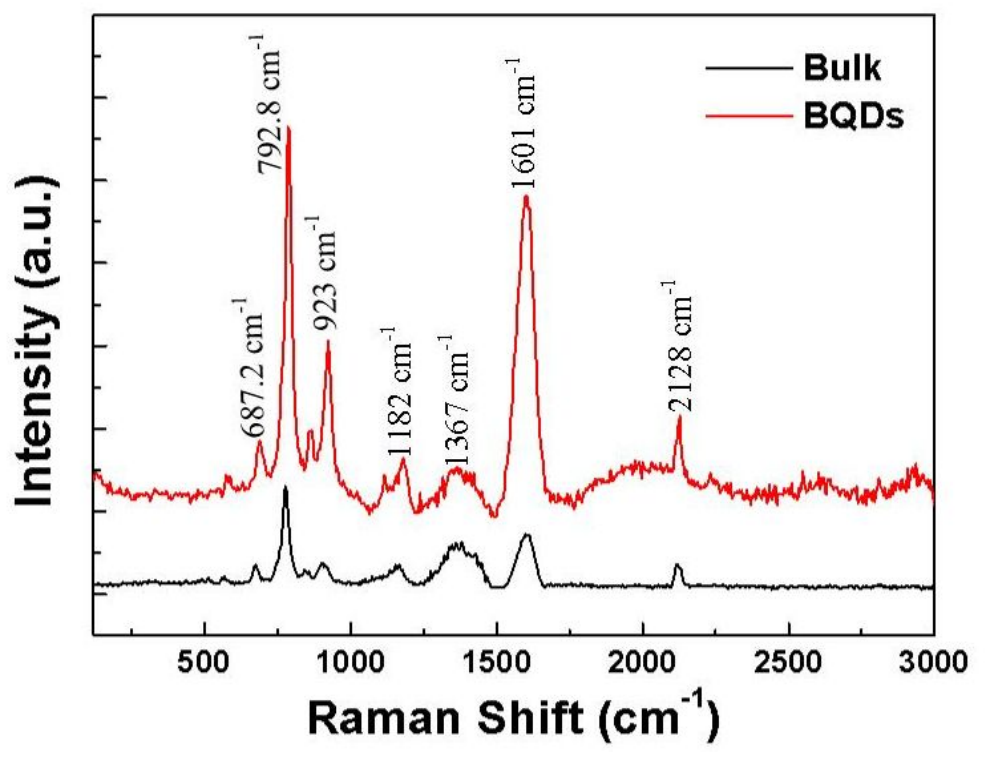

Figure S4. Raman spectra of the BQDs and the bulk counterpart. 

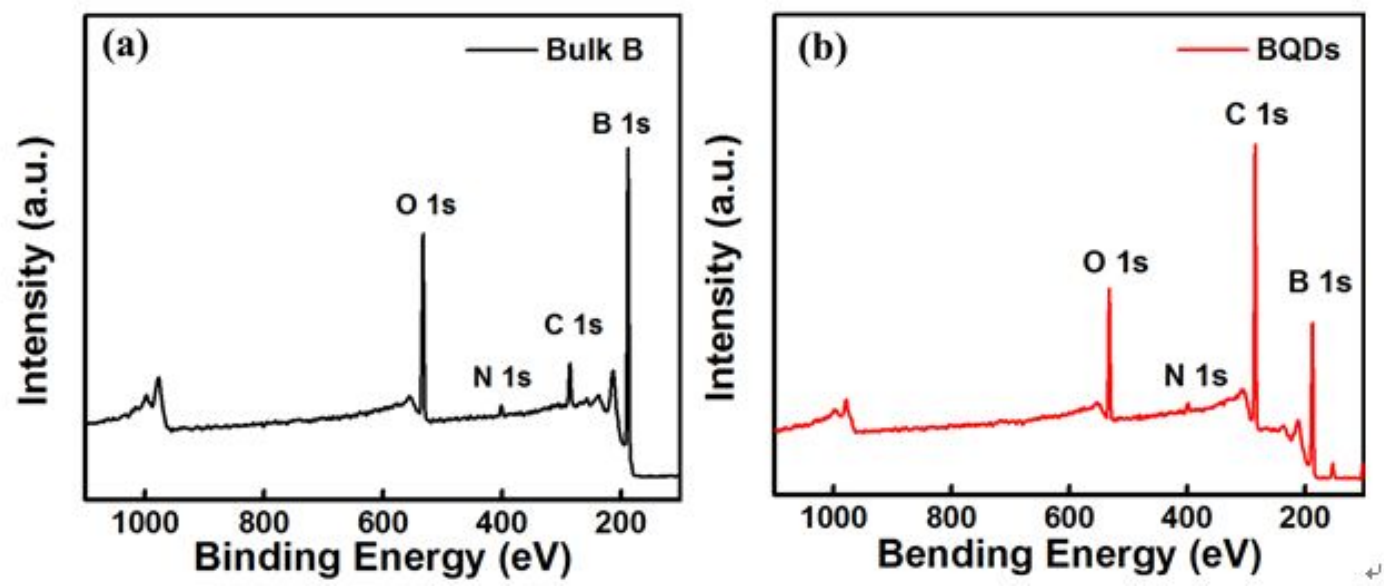

Figure S5. XPS survey-scan spectra of the boron powder (a) and the BQDs (b), respectively. It is clearly detected that four peaks, corresponding to $B, C$, $\mathrm{N}$ and $\mathrm{O}$ element, respectively. In contrast to $\mathrm{B}$ atomic ratio of the boron powder, it is noted that the content of the boron element in the BQDs is reduced, which can be attributed to impurities from the external environments and organic solvent acetontrile during the probe ultrasonic process. 

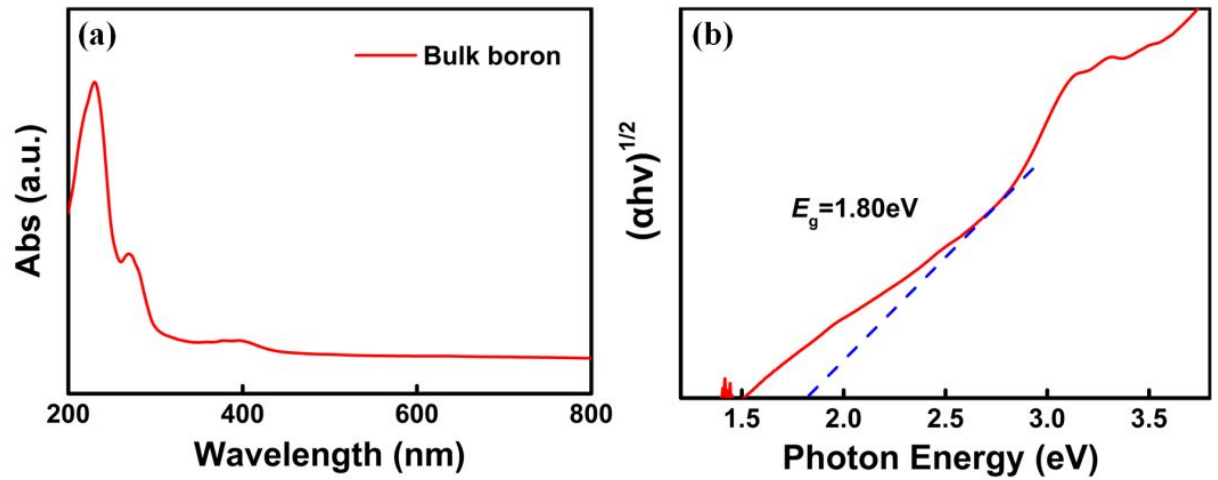

Figure S6. Optical property of the bulk boron. (a) UV-Vis spectrum of the boron powder. (b) $(\alpha h v)^{2}$ as a function of $h v$ for band gap determination. 


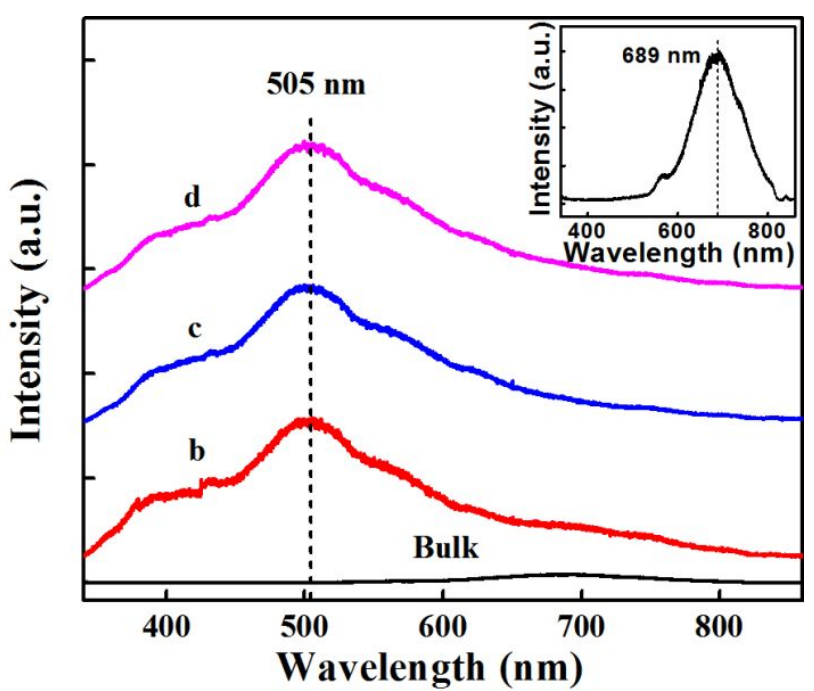

Figure S7. PL spectra of the BQDs in different batches. The numbers b, c, and d in PL are obtained by testing the BQDs of Figure S2b-d, respectively. The PL spectrum of the bulk boron is used as a reference. 

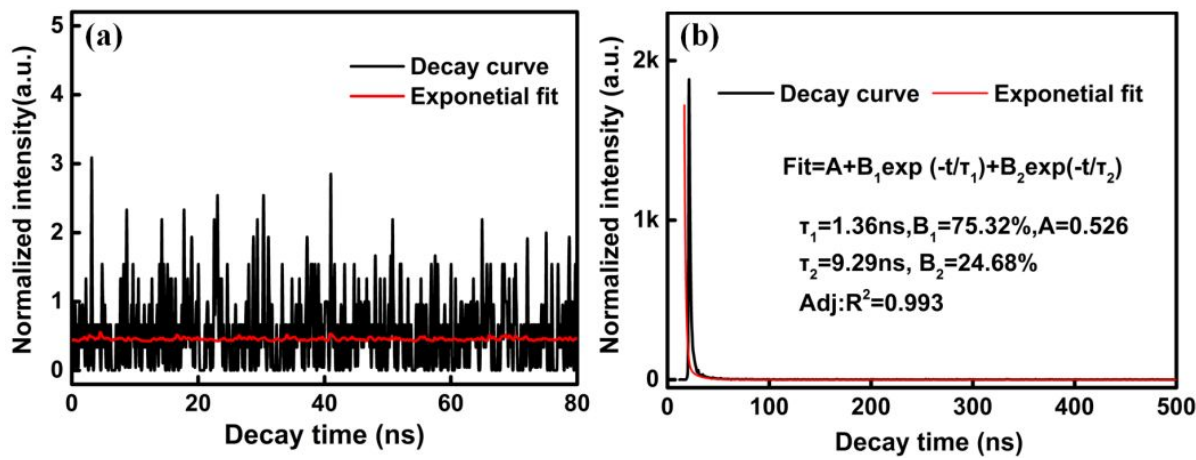

Figure S8. Time-resolved PL (TRPL) spectra of the boron powder and the BQDs. (a) TRPL spectrum of the boron powder. (b) TRPL spectrum of the BQDs-1. Inset shows the lifetime data and the parameter generated by the exponential fitting. According to the TRPL analysis of the BQDs, the recombination lifetime of the intrinsic state is shorter than the defect states, and the recombination times were 1.36 and $9.29 \mathrm{~ns}$, respectively. The TRPL spectrum of the boron powder is close to no recombination lifetime. 

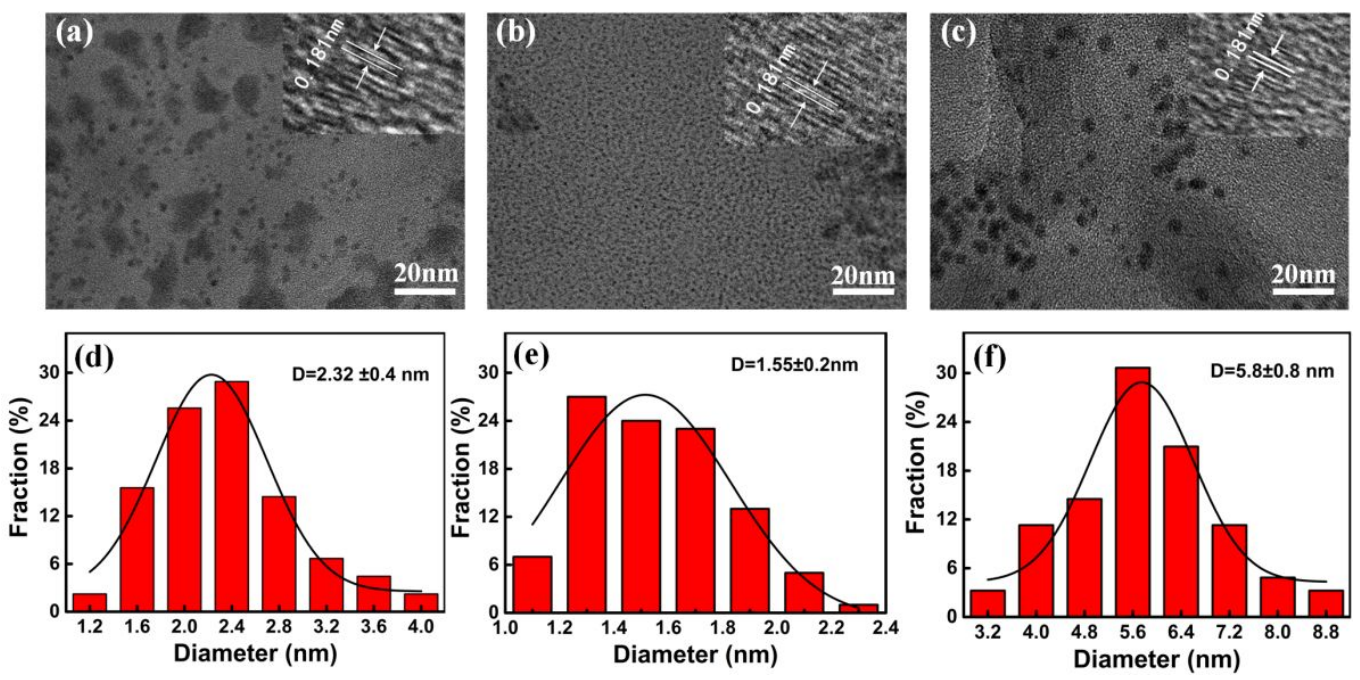

Figure S9. TEM images and the corresponding diameter distribution of the BQDs prepared under differently ultrasonic conditions. (a) TEM image of BQDs-2 synthesized at $400 \mathrm{~W}$ for $2 \mathrm{~h}$. (b) TEM image of BQDs-3 synthesized at $550 \mathrm{~W}$ for $1 \mathrm{~h}$. (c) TEM image of BQDs-3 synthesized at $400 \mathrm{~W}$ for $4 \mathrm{~h}$. (d) Diameter distribution of the quantum dots acquired from (a). (e) Diameter distribution of the quantum dots acquired from (b). (f) Diameter distribution of the quantum dots acquire from (c). 

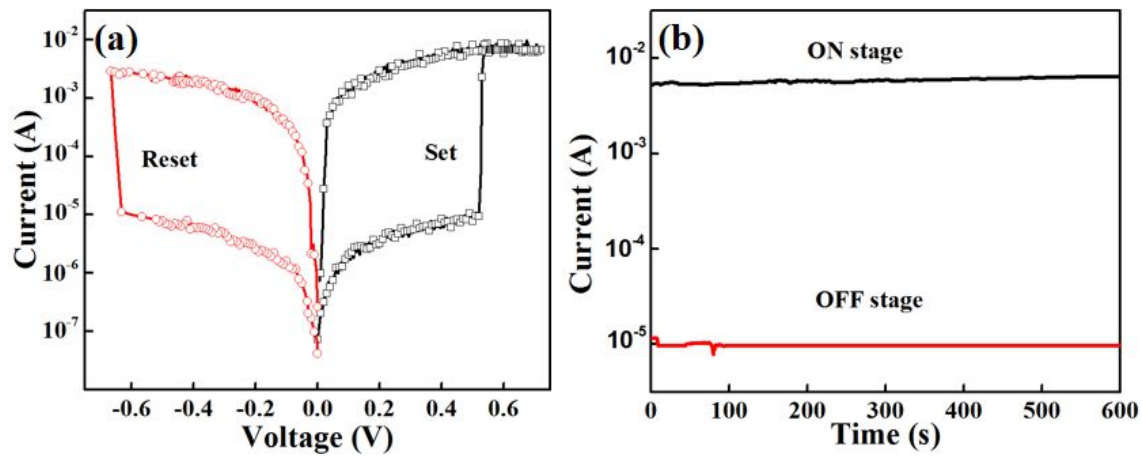

Figure S10. (a) $I-V$ curves and (b) Maintain-ability measurement of the BQDs-based nonvolatile device with a reading voltage of $0.1 \mathrm{~V}$ in another batch. 
Table S1. A table of the atomic ratios of BQDs and bulk boron powder.

\begin{tabular}{lcccc}
\hline & \multicolumn{4}{c}{ Atomic ratio (at \%) } \\
Samples & $\mathrm{B}$ & $\mathrm{C}$ & $\mathrm{N}$ & $\mathrm{O}$ \\
\hline Bulk boron & 82.16 & 6.77 & 1.06 & 10.02 \\
BQDs & 49.08 & 41.42 & 5.31 & 4.09 \\
\hline
\end{tabular}

